

\title{
Les syndromes paranéoplasiques en rhumatologie
}

\section{Paraneoplastic syndromes in rheumatology}

\author{
Fatima Belaziz, Wafae Rachidi, Kawtar Nassar, Saadia Janani, Ouafa Mkinsi
}

Service de Rhumatologie, $\mathrm{CHU}$ Ibn Rochd, Casablanca - Maroc.

\section{Correspondance à :}

Fatima BELAZIZ

belazizfatima@hotmail.fr

DOI :https://doi.org/10.48087/ B]MSra.2016.3203

Il s'agit d'un article en libre accès distribué selon les termes de la licence Creative Commons Attribution International License (CC BY 4.0), qui autorise une utilisation, une distribution et une reproduction sans restriction sur tout support ou format, à condition que l'auteur original et la revue soient dûment crédités.

\section{RÉSUMÉ}

Les syndromes paranéoplasiques sont un groupe d'affections associées à des tumeurs malignes indépendamment reliés à leurs sièges ou à leurs tailles. L'étiopathogénie incrimine des mécanismes immunitaires humoraux ou cellulaires à l'encontre des cellules tumorales. Les syndromes paranéoplasiques en rhumatologie sont rares, intéressant essentiellement les articulations, les muscles, les os, les fascias ou les vaisseaux. L'apparition de signes rhumatologiques peut précéder ou faire suite au diagnostic d'un cancer, L'intervalle entre les deux souvent ne dépasse pas plus de 3 ans, leur connaissance permet d'orienter la prise en charge de ces malades qui doit être essentiellement carcinologique.

Mots-clés : Syndrome paranéoplasique ; rhumatisme inflammatoire ; cancer.

\begin{abstract}
Paraneoplastic syndromes are a group of disorders associated with malignant diseases, particularly independent from the location and the size of the tumor. Etiopathogeny may be a consequence of humoral or cellular immune mechanisms directed against tumor cells. Paraneoplastic syndromes in rheumatology, which are rare, arise in joints, muscles, bones, fasciae or vessels. The appearance of rheumatological signs may precede or follow the diagnosis of cancer, the interval between both affections does not usually exceed 3 years, and their knowledge can guide the management of these patients that should be essentially aimed at the control of the neoplasm.
\end{abstract}

Keywords: Paraneoplastic syndrome ; Rheumatic Diseases ; cancer.

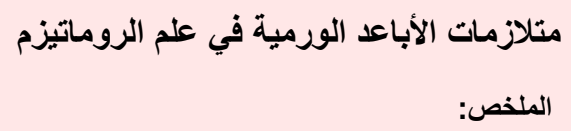

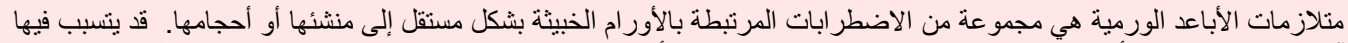

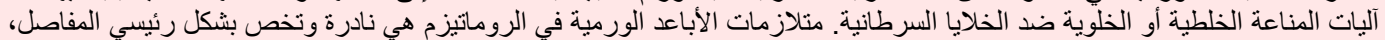



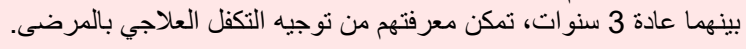
الكلمات المفتاحية: متلازمة الأباعد الورمية، الروماتيزم الالتهابي، السرطان.

\section{INTRODUCTION}

Le syndrome paranéoplasique est présent chez $10 \%$ des patients cancéreux, il regroupe un ensemble de manifestations liées à une tumeur, sans rapport anatomique direct avec elle, non expliquées par des métastases et disparaissant après cure tumorale. Rapporté pour la premièrer fois par Stertz en 1916 (polymyosite / cancer gastrique), les manifestations rhumatologiques des syndromes paranéoplasiques sont rares, leur prévalence est peu étudiée, de l'ordre de 2,65\% dans une étude portant sur 3544 cancéreux [1].

Les syndromes paranéoplasiques rhumatismaux et systémiques peuvent se classer en articulaires, neuromusculaires, cutanés, vasculaires et divers. Un rhumatisme inflammatoire paranéoplasique peut simuler une polyarthrite rhumatoïde (PR), une polyarthrite aiguë oedémateuse bénigne du sujet âgé (RS3PE) ou une pseudopolyarthrite rhizomélique (PPR). L'intervalle entre l'apparition des signes rhumatologiques et le diagnostic de la tumeur associée ne dépasse pas souvent les 3 ans, leur connaissance permet une orientation adéquate de ces patients se qui permet la détection précoce du cancer. Le traitement à visée rhumatologique est symptomatique, le traitement curatif est celui de la tumeur sous- jacente. On a exclu dans cette mise au point l'hypercalcémie paranéoplasique et les tumeurs survenant au cours de l'évolution d'une maladie autoimmune.

\section{IMMUNOPATHOGÉNIE DES SYNDROMES PARANÉOPLASIQUES RHUMATOLOGIQUES}

Deux mécanismes sont suggérés. Le premier est lié à l'effet direct de médiateurs autocrines ou paracrines (cytokines, hormones, peptides, facteurs de croissance...) libérés par les cellules tumorales qui génèrent un processus inflammatoire dans les tissus cibles. Le deuxième correspond à une réaction d'hypersensibilité due, soit à l'expression par la tumeur d'antigènes exprimés par les cellules/ 
tissus ciblés par la maladie auto-immune, soit à la libération d'antigènes intracellulaires, incluant des protéines associées aux acides nucléiques, à partir des cellules tumorales apoptotiques. Cette dysfonction du système immunitaire stimule la production d'auto anticorps et de lymphocytes cytotoxiques. Il en résulte la production, non seulement d'auto anticorps anti-nucléaires voire anti-ADN double brin, mais aussi de toute une variété d'auto anticorps dirigés contre des antigènes tissulaires dans le sérum des patients ayant ces manifestations paranéoplasiques. Ces 2 mécanismes peuvent être associés.

\section{RHUMATISMES INFLAMMATOIRES PARANÉOPLASIQUES}

\section{Polyarthrites paranéoplasiques}

Rares, avec seulement 8 séries publiées (1984-2014) intéressent 121 patients dont le $1 / 3$ avait une hémopathie maligne, les tumeurs solides les plus fréquentes étaient des adénocarcinomes du poumon et du sein. Elles précèdent le diagnostic du cancer de 10 mois en moyenne [2]. Séronégatives le plus souvent, les facteurs rhumatoïdes et ACPA sont retrouvés dans $27,2 \%$ et $10,7 \%$ des cas respectivement, dans l'étude récente de kisacik [3].

Apanage du sujet âgé de plus de 50 ans. Elles se caractérisent cliniquement par un début rapide et explosif, une altération de l'état général et la résistance aux traitements usuels (corticoïdes / immunosuppresseurs), l'atteinte est asymétrique épargnant les poignets et les articulations des mains, non déformante, non destructrice. La régression de l'arthrite après traitement antitumoral permet de retenir le diagnostic rétrospectivement.

\section{La polyarthrite aiguë œdémateuse bénigne du sujet âgé ou RS3PE syndrome (Remitting seronegative symmetrical synovitis with pitting edema)}

Sa physiopathologie incrimine le VEGF ainsi que l'IL-6. Des taux élevés de MMP3 (métalloprotéinase 3), impliqué dans la destruction cartilagineuse au cours des rhumatismes, a été retrouvés dans le sérum de patients présentant un RS3PE, il est aussi présent à des taux élevés chez des patients porteurs de néoplasie [4].

Sa prévalence dans la littérature varie entre $6 \%$ et $24,7 \%$ [5]. Survenant le plus souvent au cours d'un adénocarcinome prostatique ou gastro-intestinal. La description clinique est classique, il s'agit d'une polyarthrite survenant au-delà de 60 ans d'installation brutale, symétrique non érosive non déformante touchant les petites articulations des mains avec un œdème du dos des mains et des pieds, des ténosynovites des extenseurs/fléchisseurs. Sans autoanticorps, le caractère paranéoplasique est suspecté en cas de corticorésistance.

\section{Pseudopolyarthrite rhizomélique.}

Les pathologies tumorales observées sont généralement des syndromes myéloprolifératifs (myélome multiple) et le plus souvent un syndrome myélodysplasique suspecté devant une anémie pernicieuse, une thrombopénie ou une neutropénie [6]. Le caractère paranéoplasique est suspecté devant des atypies cliniques dont un âge inférieur à 50 ans, une atteinte asymétrique voire unilatérale sur les sites habituels, une VS anormalement basse (< $40 \mathrm{~mm} 1$ ère heure) ou très élevée $(>$ 100 ) et surtout la corticorésistance [7]. Ainsi devant une PPR corticorésistante avec des anomalies hématologiques, il convient de faire un frottis sanguin. Le Cancer solide est une situation exceptionnelle. Autrement il n'y a pas d'argument pour rechercher en première intention un cancer chez un patient atteint de PPR. D'autres auteurs ne retrouvent pas d'association entre les deux pathologies [8].

\section{Rhumatisme de Jaccoud paranéoplasique}

Décrit au cours des rhumatismes articulaires, surtout dans le lupus systémique. Quelques cas rapportés au cours de cancers des poumons [9], et de cancer hépatique [10]. Sa physiopathologie demeure méconnue.

\section{ARTHRITE PARANÉOPLASIQUE ET MANIFESTATIONS CUTANÉES}

\section{Syndrome fasciite palmaire, polyarthrite}

Secondaire à un adénocarcinome dans $74 \%$ des cas dont 37 $\%$ sont ovariens. Il est 4 fois plus fréquent chez la femme. Il précède le diagnostic du cancer en moyenne de 6 mois, qui est dans plus des $2 / 3$ des cas à des stades avancés métastatiques [2]. Il se manifeste au début par l'association d'enraidissement des articulations des mains, d'œdème palmaire et d'un épaississement nodulaire de l'aponévrose palmaire. A la phase d'état s'installe un tableau sclérodermiforme fait d'une rétraction des gaines synoviales, d'une flexion irréductible des doigts mais pas de phénomène de Raynaud.

La polyarthrite est bilatérale et symétrique, touchant les IP, MCP, poignets, coudes, et genoux, S'y associe une capsulite rétractile de l'épaule (syndrome épaule-main), une extension aux pieds dans un $1 / 4$ des cas [11]. Les AAN sont négatifs dans $92 \%$ des cas. La radiographie des mains retrouve une déminéralisation diffuse, et des petites érosions articulaires (carpe, MCP et IP) sont possibles. La capillaroscopie est normale.

Les anti-inflammatoires non stéroïdiens, corticoïdes, immunosuppresseurs (Cs DMARDs) ont des résultats décevants, le traitement de la tumeur diminue l'inflammation, mais la flexion irréductible des doigts demeure irréversible. Récemment l'adalimumab a été utilisé en 2 ème ligne chez un patient traité par association adalimumab/MTX, il y avait une diminution modeste de l'inflammation au prix d'une rechute de la maladie cancéreuse [12].

\section{Panniculite - arthrite}

Elle accompagne les cancers pancréatiques. Cliniquement, on retrouve des nodules sous-cutanés rouges et inflammatoires, localisés sur les jambes, les cuisses et les fesses, qui se liquéfient et laissent sourdre un liquide jaunâtre stérile. L'arthrite touche en règle les poignets, les MCP, les genoux et les chevilles. Des douleurs osseuses des os longs est possible [13]. L'histologie est pathognomonique : il s'agit de lésions de nécrobiose aseptique surmontée par un infiltrat inflammatoire. Le taux plasmatique des enzymes pancréatiques (lipase/amylase/trypsine) est élevé. Une hyperéosinophilie sanguine est plus fréquemment observée en cas de cancer. 
La corticothérapie est en général inefficace, la symptomatologie régresse sous traitement du cancer pancréatique.

\section{Réticulohistiocytose multicentrique paranéoplasique}

C'est une histiocytose non langerhansienne, néoplasique dans environ 15 à $28 \%$ des cas (ADK) avec un Sex-ratio H/F de $1 / 3$, l'âge de début se situe entre 40 et 50 ans. Une polyarthrite séronégative mimant une PR est inaugurale dans $2 / 3$ des cas (bilatérale, symétrique touchant les mains, les poignets), destructrice et mutilante 1 fois/2, avec atteinte des IPD. Les manifestations cutanées sont faites d'éruption papulonodulaire, symétrique sur les faces dorsales des doigts et dans les zones juxta-articulaires en aspect caractéristique en lit de corail [14].

L'étude histopathologie d'une biopsie cutanée ou synoviale permet le diagnostic positif, elle montre une infiltration histiocytaire et des cellules géantes multinucléées au cytoplasme " en verre dépoli ». L'évolution se fait par poussées. Le traitement est la cure de néoplasie, la corticothérapie peut être utilisée à faible dose.

\section{SYNDROMES NEURO-MUSCULAIRES PARANÉOPLASIQUES}

\section{Polymyosite/dermatomyosite}

De nombreuses études confirment l'augmentation du risque de cancer au cours des dermato/polymyosites; il est au moins 3 fois supérieur à celui de la population générale.

Le risque relatif pour la dermatomyosite est de 3,4 à 4,4 et seulement de 1,7 à 2,1 pour la polymyosite. L'incidence de l'association cancer/DM varie entre $7 \%$ et $30 \%$. Les cancers les plus fréquemment retrouvés associés aux dermatomyosites sont les adénocarcinomes (ovaire, estomac, pancréas), puis les lymphomes. Au cours des polymyosites, ce sont les lymphomes non hodgkiniens et à moindre degré les cancers du poumon et de la vessie. Les myosites nodulaires sont exceptionnellement associées à un cancer ou à un lymphome [15].

La physiopathologie demeure inconnue, la production d'autoanticorps liée à l'hyper-expression dans le tissu tumoral d'antigènes spécifiques des fibres musculaire a été avancée comme hypothèse. Les anticorps anti-p155 ont été isolés au cours des dermatomyosites paranéoplasiques avec une sensibilité de $78 \%$ et une spécificité de $89 \%$ [16], ils peuvent être un excellent argument diagnostique [17]. Les anticorps anti-Jo-1 ou anti-Mi2 par contre représentent un facteur protecteur [18]. En faveur du caractère paranéoplasique, on note un début rapide, une atteinte musculaire/cutanée sévère, le caractère nécrotique des lésions cutanées, l'âge élevé, et la corticorésistance. Aussi, une élévation plus importante des CPK et un syndrome inflammatoire plus marqué sont évocateurs. Une atteinte interstitielle respiratoire associée à un risque moindre. Devant une myopathie inflammatoire, il est nécessaire de rechercher une tumeur occulte par un examen clinique complet avec touchers pelviens, les explorations de première intention comportent un Hémocult, un scanner thoraco-abdominopelvien, une mammographie et une échographie pelvienne (chez la femme). Une endoscopie si point d'appel clinique. La place du TEP-scanner couplé aux Ac anti-p155 est à déterminer [19]. Ces explorations doivent être refaites tous les 6 mois, il n'est pas recommandé de les poursuivre au-delà 3 années de suivi, la néoplasie survient généralement au cours des 3 premières années d'évolution [20]. Le traitement symptomatique comporte la corticothérapie généralement efficace mais parfois les manifestations cutanées résistent. Dans l'étude de Andras [15], le taux de rémission après traitement carcinologique est de $53 \%$, le taux de survie à 5 ans est de $56 \%$ contre $92 \%$ pour les formes idiopathiques. Le pronostic global est péjoratif.

\section{Le Syndrome myasthéniforme de Lambert et Eaton (LEMS)}

Sa prévalence est estimée à 3,42 cas par million d'habitants. Paranéoplasique dans $60 \%$ des cas, le plus souvent associé à un cancer pulmonaire à petites cellules découvert après 1 à 2 années d'évolution. Il est dû à une réduction du relargage d'acétylcholine au niveau de la transmission neuromusculaire par des anticorps dirigés contre des canaux calciques voltagedépendants présynaptiques (anti-VGCC), présent dans 85-90 $\%$ des cas. L'anticorps anti-Sox1 secrété par les cancers pulmonaires à petites cellules est un marqueur possible du LEMS paranéoplasique avec une sensibilité de $67 \%$ et une spécificité de $95 \%$ [21].

Cliniquement, il se manifeste par une fatigabilité excessive aux efforts, une faiblesse musculaire pelvienne, des signes dysautonomiques, une hyporéflexie. L'électromyogramme retrouve un bloc neuromusculaire présynaptique.

Titulaer a proposé un score prédictif de la présence d'un carcinome bronchopulmonaire à petites cellules (CBPC) au moment du diagnostic de LEMS, appelé Delta-score, La probabilité d'un CBPC varie de 0 à $2,6 \%$ pour un Delta-score entre 0 et 1 , de 83,9 à $100 \%$ pour un score de 3 à 6 [22]. Le traitement du cancer est la première étape de prise en charge. Le traitement symptomatique inclut la 3-4 diaminopyridine +/- associée au inhibiteurs de l'acétylcholine-estérase, dans les formes sévères les corticoïdes, les Immunoglobulines intraveineuses et en 2 ème intention les immunosuppresseurs en association (cyclophosphamide, azathioprine), peuvent être utilisés [23].

\section{Le syndrome de l'homme raide}

Il s'agit plus d'une affection immunologique plutôt que néoplasique. Elle se caractérise par une rigidité progressive diffuse, axiale et aux membres inférieurs associée à des spasmes déclenchés par un contact ou une contraction volontaire. Les affections malignes rapportées au cours du syndrome de la personne raide sont surtout le cancer du sein, et dans quelques cas le cancer du poumon à petites cellules, la maladie de Hodgkin, le thymome. Les anticorps anti glutamic acid decarboxylase ou GAD se rencontrent volontiers dans la forme auto-immune et plus rarement dans la forme paranéoplasique.

\section{ATTEINTES VASCULAIRES PARANÉOPLASIQUES}

\section{Le syndrome de Raynaud paranéoplasique}

Chez certains patients, en général d'âge $>50$ ans, un syndrome de Raynaud souvent sévère peut être en relation avec un cancer des poumons, du tractus digestif, de l'ovaire ou du sein, du pancréas, du rein ou bien une hémopathie. 


\section{L'érythromélalgie paranéoplasique}

Elle se traduit par des accès douloureux accompagnés de gonflement et de rougeur des téguments siégeant aux extrémités, surtout aux pieds. Les crises s'atténuent au froid et sous l'action de l'aspirine. Une érythromélalgie est possiblement liée à une polyglobulie de Vaquez ou une thrombocytémie essentielle.

\section{La nécrose digitale paranéoplasique}

Une nécrose digitale paranéoplasique peut révéler une tumeur maligne, en particulier bronchopulmonaire, digestive ou rénale. Le mécanisme en est une vascularite paranéoplasique, un spasme artériel (proche du phénomène de Raynaud) et des microthromboses.

\section{Vascularite paranéoplasique}

La néoplasie associée est dans les $2 / 3$ des cas une hémopathie maligne notamment une leucémie à tricholeucocytes avec une fréquence de 1,5 à $19 \%$, quelques cas de myélome à IgA ont été rapportés. L'expression clinique est souvent celle d'une vascularite leucocytoclasique, ou une périartérite noueuse (PAN) [24]. Une maladie de Horton paranéoplasique est aussi décrite [25]. L'association " vascularite-tumeur solide » est exceptionnelle, il s'agit de cancers pulmonaires, digestifs et uro-génitaux. La forme clinique est celle d'un purpura rhumatoïde dans $63 \%$ des cas, d'une granulomatose de Wegener en cas de cancer du rein [26].

La particularité des vascularites paranéoplasiques est l'absence de consommation du complément et l'absence d'auto-anticorps, les ANCA sont retrouvés dans moins de $5 \%$ des cas. Seulement $5 \%$ des patients présentant une vascularite sont porteurs de néoplasie [27]. Actuellement, il n'y a aucune recommandation pour les explorations à la recherche d'une néoplasie au cours des vascularites systémiques.

\section{CONNECTIVITES}

\section{Sclérodermie paranéoplasique}

Le caractère paranéoplasique est suspecté devant un début tardif (> 50 ans), un syndrome de Raynaud d'installation brutale, une sclérose s'étendant au tronc, le risque relatif de développer un cancer dans l'étude de Bonifazi et col est de 1,75 [28]. Les associations néplasie/ sclérodermie décrites sont les cancers pulmonaires, mammaires, les lymphomes et le myélome ostéo-condensant (POEMS) [29]. La survenue d'un cancer est plus fréquente chez les patients ayant des anticorps anti-RNA polymérase III avec un risque multiplié par 6 [30]. La régression de la symptomatologie après traitement de la tumeur sous-jacente a été rapportée dans quelques cas [31].

\section{Lupus like-syndrome}

La relation entre ces deux pathologies est incertaine. Des associations avec un cancer de l'ovaire, du sein et une leucémie à tricholeucocytes ont été rapportées, décrites, avec une rémission complète après traitement carcinologique [29].

\section{Pseudo-maladie de still paranéoplasique}

L'existence d'un processus tumoral est un critère d'exclusion de la MSA. Néanmoins, des observations de manifestations évocatrices d'une MSA précédant le diagnostic d'une néoplasie et disparaissant après son traitement sont rapportées [32].

\section{Polychondrite atrophiante}

Les cancers décrits sont principalement des syndromes myélodysplasiques (anémies réfractaires (60\%). La fréquence des SMD dans les séries de polychondrites varie entre $9 \%$ et $28 \%$, avec une prédominance masculine et un début plus tardif, ainsi $40 \%$ des sujets de plus de 60 ans présentant une polychondrite ont une myélodysplasie, le traitement de la polychondrite par corticoïdes, dapsone ou immunosuppresseurs est peu efficace. Le pronostic est mauvais avec un décès dans $50 \%$ des cas principalement de complications infectieuses (respiratoires) [6].

\section{AUTRES PRÉSENTATIONS CLINIQUES PARANÉOPLASIQUES}

\section{La goutte articulaire et rénale}

Elle peut se développer chez des patients souffrant d'une leucémie, d'une polyglobulie de Vaquez, d'une thrombocytémie essentielle, d'un lymphome, d'un myélome, et plus rarement d'un carcinome, avant ou après la mise en route de la chimiothérapie [33].

\section{L'arthropathie amyloïde}

Elle se rencontre essentiellement au cours du myélome multiple [34]. L'algodystrophie paranéoplasique peut s'associer à des tumeurs malignes de différents types, en particulier le cancer épidermoïde du poumon mais aussi les cancers du côlon, du pancréas, de l'ovaire ou des leucémies myéloïdes.-

\section{Ostéoarthropathie hypertrophiante pneumique de Pierre Marie (OAH)}

L'OAH de Pierre Marie prenant également le nom de pachydermopériostose est un syndrome clinique et radiologique associant un hippocratisme digital, une périostose des os longs et une polyarthrite subaigue ou chronique, secondaire dans $90 \%$ à une néoplasie bronchique non à petites cellules. La prévalence de l'OAH pneumique de Pierre Marie est estimée entre 3 et $5 \%$ de l'ensemble des $\mathrm{OAH}$ secondaires. Du fait de l'augmentation de l'incidence du cancer pulmonaire chez la femme, le sex-ratio a manifestement changé durant ces dernières années et estimé actuellement à 11 hommes pour cinq femmes [35]. Des hypothèses pathogéniques ont été proposées dans la survenue de l'OAH, la plus pertinente est celle du shunt droitgauche. En effet, cette anomalie anatomique pourrait être responsable de la présence dans la grande circulation de mégacaryocytes incomplètement clivés. L'agrégation périphérique des plaquettes dans le lit capillaire serait à l'origine d'une libération de facteurs de croissance, inducteurs de la formation de la matrice extracellulaire (PDGF et TGF $\beta 1$ ) ou vasodilatateurs (VEGF). D'autres mécanismes ont été suggérés dans la physiopathologie de l'OAH telle l'hormone de croissance interagissant avec 
d'autres facteurs vasodilatateurs associant l'hypertonie vagale, la ferritine et les prostaglandines (PGE2 et PGF2) [36]. Cliniquement, l'OAH est souvent le syndrome révélateur lorsque la néoplasie pulmonaire évolue lentement et silencieusement, elle se manifeste comme un ensemble syndromique associant un syndrome acromélique comportant un hippocratisme digital, une hypertrophie des parties molles et des troubles vasomoteurs, un syndrome articulaire avec des arthralgies ou arthrites et un syndrome osseux comprenant une périostose engainante.

\section{La fasciite de Shulman (Fasciite à éosinophilie)}

Il s'agit de fasciites des extrémités et du tronc associées à des rétractions articulaires et à une éosinophilie sanguine avec hyper gammaglobulinémie. Des formes familiales ont été rapportées au cours du cancer mammaire. Le tableau de fasciite précède habituellement le cancer de plusieurs mois (médiane $1 \mathrm{an}$ ), la prédominance féminine est habituelle. Il n'y a pas de bonne réponse à la corticothérapie et la régression est inconstante après le traitement de la tumeur

\section{CONCLUSION}

Les rhumatismes inflammatoires paranéoplasiques sont rares, leur prévalence ne dépasse pas 3\%, l'étiopathogénie est inconnu et toujours débattue. Certaines atypies cliniques et paracliniques orientent le diagnostic mais c'est la disparition de ces manifestations après traitement antitumoral qui permet rétrospectivement le diagnostic positif.

Déclaration d'intérêts: les auteurs ne déclarent aucun conflit d'intérêt en rapport avec cet article.

\section{RÉFÉRENCES}

1. Rugienè R, Dadonienè J, Aleknavičius E, Tikuišis R, Distler J, Schett G, et al. Prevalence of paraneoplastic rheumatic syndromes and their antibody profile among patients with solid tumours. Clin Rheumatol. 2011;30(3):373-80

2. Manger B, Schett G. Paraneoplastic syndromes in rheumatology. Nat Rev Rheumatol. 2014 (19): 65-7

3. Kisacik B1, Onat AM, Kasifoglu T, Pehlivan Y, Pamuk ON, Dalkilic E et al Diagnostic dilemma of paraneoplastic arthritis: case series. Int J Rheum Dis. 2014 ;17(6):640-5

4. Origuchi T, Arima K, Kawashiri SY, Tamai M, Yamasaki S, Nakamura $H$, et al. High serum matrix metalloproteinase 3 is characteristic of patients with paraneoplastic remitting seronegative symmetrical synovitis with pitting edema syndrome. Mod Rheumatol. 2012;22(4):584-8

5. Kimura M, Tokuda $\mathrm{Y}$, Oshiawa $H$, Yoshida K, Utsunomiya $M$, Kobayashi T, Clinical characteristics of patients with remitting seronegative symmetrical synovitis with pitting edema compared to patients with pure polymyalgia rheumatica. I Rheumatol. 2012;39(1):148-53

6. Fain O1, Braun T, Stirnemann J, Fenaux P.Systemic and autoimmune manifestations in myelodysplastic syndromes.Rev Med Interne. 2011;32(9):552-9.
7. Naschitz JE. Rheumatic syndromes: clues to occult neoplasia. Curr Opin Rheumatol. 2001;13(1):62-6.

8. Myklebust G, Wilsgaard T, Jacobsen BK, Gran JT. No increased frequency of malignant neoplasms in polymyalgia rheumatica and temporal arteritis. A prospective longitudinal study of 398 cases and matched population controls. J Rheumatol. 2002;29(10):2143-7.

9. Johnson JJ, Leonard-Segal A, Nashel DJ. Jaccoud's-type arthropathy: an association with malignancy. J Rheumatol. 1989;16(9):1278-80.

10. Tekaya R, Ben Abdelghani K, Abdelmoula L, et al. Arthropathie de Jaccoud paranéoplasique. Revue du Rhumatisme 2007 ; 74 : 10391208

11. Masson C, Bouvard B, Audran M. Manifestations rhumatologiques paranéoplasiques en dehors de l'ostéoarthropathie hypertrophiante. EMC - Rhumatologie-Orthopédie2005;2(5): 501-9

12. Nadal R, McMahan ZH, Antonarakis ES. Paraneoplastic palmar fasciitis and polyarthritis syndrome in a patient with advanced prostate cancer. Clin Genitourin Cancer. 2013;11(4):15-23.

13. Ashouri JF, Daikh DI. Rheumatic manifestations of cancer. Rheum Dis Clin North Am. 2011;37(4):489-505

14. Nicol C, Quereux G, Renaut JJ, Renac F, Dreno B. Paraneoplastic multicentric reticulohistiocytosis. Ann Dermatol Venereol. 2011;138(5):405-8

15. András C1, Ponyi A, Constantin T, Csiki Z, Szekanecz E, Szodoray P, et al. Dermatomyositis and polymyositis associated with malignancy: a 21-year retrospective study. J Rheumatol. 2008; 35(3):438-44.

16. Trallero-Araguás E, Rodrigo-Pendás JÁ, Selva-O'Callaghan $A$, Martínez-Gómez X, Bosch X, Labrador-Horrillo M. Usefulness of antip155 autoantibody for diagnosing cancer-associated dermatomyositis: a systematic review and meta-analysis. Arthritis Rheum. 2012;64(2):523-32.

17. Szankai Z, Nagy-Vincze M, Bodoki L, Jakab A, Betteridge Z, Dankó K. Risk factors for cancer in patients with myositis. Clinical, immunological characteristics and the role of the anti-p155/140 antibody. Orv Hetil. 2014;155(36):1437-44.

18. Scalapino KJ, Thomas CR Jr. Paraneoplastic and cancer treatmentrelated rheumatic disorders. Rheum Dis Clin North Am. 2011;37(4):43-5

19. Selva-O'Callaghan A, Trallero-Araguás E, Grau-Junyent JM, LabradorHorrillo M. Malignancy and myositis: novel autoantibodies and new insights. Curr Opin Rheumatol. 2010;22(6):627-32.

20. Buchbinder R, Forbes $A$, Hall $S$, Dennett $X$, Giles $G$. Incidence of malignant disease in biopsy-proven inflammatory myopathy. A population-based cohort study. Ann Intern 2001;134:1087-95.

21. Didelot A, Honnorat J. Paraneoplastic neurological syndromes. Rev Med Interne. 2011;32(10):605-11

22. Titulaer MJ, Maddison P, Sont JK, Wirtz PW, Hilton-Jones D, Klooster $R$ et al.Clinical Dutch-English Lambert-Eaton Myasthenic syndrome (LEMS) tumor association prediction score accurately predicts smallcell lung cancer in the LEMS. J Clin Oncol. 2011;29(7):902-8.

23. Boutin E, Rey C, Romeu M, Pouget J, Franques J. Favourable outcome after treatment with rituximab in a case of seronegative non-paraneoplastic Lambert-Eaton myasthenic syndrome]. Rev Med Interne. 2013;34(8):493-6.

24. Fain O, Guillevin L, Kaplan G, Sicard D, Lemaire V, Godeau P, et al. Vascularites et néoplasies : 14 observations. Ann Med Interne1991;142: 486-504.

25. Ungprasert P, Sanguankeo A, Upala S, Knight EL. Risk of malignancy in patients with giant cell arteritis and polymyalgia rheumatica: a systematic review and meta-analysis. Semin Arthritis Rheum. 2014; 44(3):366-70. 
26. Greer JM, Longley S, Edwards NL, Elfenbein GJ, Panush RS. Vasculitis associated with malignancy. Experience with 13 patients and literature review. Medicine (Baltimore) 1988;67: 220-30.

27. Pasquet $F$, Pavic $M$, Ninet J, Hot A. Auto-immune diseases and cancers. Second part: Auto-immune diseases complicating cancers and their treatment. Rev Med Interne. 2014;35(10): 656-63.

28. Bonifazi M, Tramacere I, Pomponio G, Gabrielli B, Avvedimento EV, La Vecchia $C$ et al. Systemic sclerosis (scleroderma) and cancer risk: systematic review and meta-analysis of observational studies. Rheumatology. 2013 ;52(1):143-54.

29. Racanelli V, Prete M, Minoia C, Favoino E, Perosa F. Rheumatic disorders as paraneoplastic syndromes. Autoimmun Rev. 2008;7(5):352-8.

30. Moinzadeh P, Fonseca C, Hellmich M, Shah AA, Chighizola C, Denton $\mathrm{CP}$, et al. Association of anti-RNA polymerase III autoantibodies and cancer in scleroderma. Arthritis Res Ther. 2014;16(1):53.

31. R. Bellis, C. Francès, S. Barète, P. Senet. Régression clinique et capillaroscopique après autogreffe de moelle d'une sclérodermie systémique cutanée limitée associée à un lymphome anaplasique CD30. Annales de Dermatologie et de Vénéréologie 2014; 141, (6 7) : 446-451.
32. Liozon E, Ly KH, Vidal-Cathala E, Fauchais AL. Adult-onset Still's disease as a manifestation of malignancy: report of a patient with melanoma and literature review. Rev Med Interne. 2014;35(1):60-4.

33. Möhrenschlager M, Köhn F, Ring J. [Dermatologic manifestations of internal diseases. 12: Gout, paraneoplasias and striae distensae]. MMW Fortschritte der Medizin. 2002;144(23):44-6. 33.

34. Racanelli V, Prete M, Minoia C, Favoino E, Perosa F. Rheumatic disorders as paraneoplastic syndromes. Autoimmunity Reviews. $2008 ; 7(5): 352-8$.

35. Morel J, Deschamps V, Toussirot E, Pertuiset E, Sordet C, Kieffer P, et al. Characteristics and survival of 26 patients with paraneoplastic arthritis. Annals of the Rheumatic Diseases. 2008;67(2):244-7.

36. Masson C, Bouvard B, Audran M. Manifestations rhumatologiques paranéoplasiques en dehors de l'ostéoarthropathie hypertrophiante. EMC - Appareil locomoteur. 2006;1(1):1-6.
Cet article a été publié dans le « Batna Journal of Medical Sciences » BJMS, l'organe officiel de " l'association de la Recherche Pharmaceutique - Batna»

Le contenu de la Revue est ouvert "Open Access » et permet au lecteur de télécharger, d'utiliser le contenu dans un but personnel ou d'enseignement, sans demander l'autorisation de l'éditeur/auteur.

Avantages à publier dans BJMS :

- Open access : une fois publié, votre article est disponible gratuitement au téléchargement

Soumission gratuite : pas de frais de soumission, contrairement à la plupart des revues « Open Access »

Possibilité de publier dans 3 langues : français, anglais, arabe

- Qualité de la relecture : des relecteurs/reviewers indépendants géographiquement, respectant l'anonymat, pour garantir la neutralité et la qualité des manuscrits.

Pour plus d'informations, contacter BatnaJMS@gmail.com

ou connectez-vous sur le site de la revue : www.batnajms.com

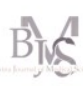

Batna Journal of



